\title{
Eunotiaceae Kützing (Bacillariophyceae) planctônicas do Sistema Lago dos Tigres, Britânia, GO, Brasil
}

\author{
Bárbara Dunck Oliveira ${ }^{1,4}$, Ina de Souza Nogueira ${ }^{2}$ e Maria da Graça Machado de Souza ${ }^{3}$
}

Recebido: 28.07.2011; aceito: 18.06 .2012

\begin{abstract}
Planktonic Eunotiaceae Kützing (Bacillariophyceae) of Sistema Lago dos Tigres, Britânia, Goiás State, Brazil). This study aimed to survey the species of Eunotiaceae Kützing family occurring in the plankton of Sistema Lago

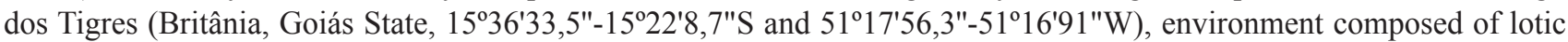
and lentic areas. Sampling was conducted between June and November 2004 at 11 sampling stations, and samples were collected through a plankton net of $25 \mu \mathrm{m}$. Sistema Lago dos Tigres presented 22 taxa, one for Actinella and 21 belonging to Eunotia. Eunotia asterionelloides was the one most common species at sample stations and was recorded in all environments. Among the taxa, 10 are cited for the first time for the midwestern region of Brazil (Eunotia deficiens, E. diodonopsis, E. intermedia, E. longicollis, E. papilio, E. pileus, E. rabenhorstiana var. elongata, E. rabenhorstii var. monodon, E. trigibba, and E. tropico-arcus).
\end{abstract}

Key words: Actinella, diatom, Eunotia, phytoplankton

RESUMO - (Eunotiaceae Kützing (Bacillariophyceae) planctônicas do Sistema Lago dos Tigres, Britânia, GO, Brasil). Este trabalho objetivou inventariar as espécies da família Eunotiaceae Kützing ocorrentes no plâncton do sistema Lago

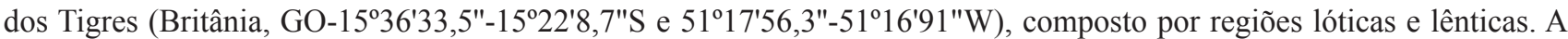
amostragem foi realizada entre junho e novembro de 2004 em 11 estações amostrais, e amostras coletadas através de rede de plâncton $25 \mu \mathrm{m}$. O sistema Lago dos Tigres apresentou 22 táxons, sendo um para Actinella e 21 para Eunotia. Eunotia asterionelloides foi o táxon mais comum, registrado em todas as estações amostrais. Entre os táxons, 10 citações são pioneiras para a região Centro-Oeste do Brasil (Eunotia deficiens, E. diodonopsis, E. intermedia, E. longicollis, E. papilio, E. pileus, E. rabenhorstiana var. elongata, E. rabenhorstii var. monodon, E. trigibba e E. tropico-arcus).

Palavras-chave: Actinella, diatomáceas, Eunotia, fitoplâncton

\section{Introdução}

A família Eunotiaceae Kützing é caracterizada por apresentar indivíduos sem nódulo central e com rafe rudimentar nos dois ápices de ambas as valvas (Round et al. 1990). Para ambientes aquáticos dulcícolas os gêneros mais comuns são Eunotia Ehrenberg e Actinella Lewis, com ampla distribuição nas regiões tropicais e subtropicais do planeta, e são conhecidos por serem diatomáceas acidófilas e predominantemente de hábito perifítico, que também podem compor o fitoplâncton (Round et al. 1990).

O gênero Actinella descrito para a América do Sul, para a região da Australásia e Madagascar, possui em torno de 60 espécies (Metzeltin \& Lange Bertalot 1998, Kocioleck et al. 2001, 2007, Sabbe et al. 2001). O gênero Eunotia possui cerca de 300 táxons específicos e infraespecíficos (Metzeltin et al. 2005) e cerca de 80 já foram registrados no Brasil (Ludwig \& Bigunas 2006). Recentemente, para esse

1. Universidade Estadual de Maringá - Programa de Pós-Graduação em Ecologia de Ambientes Continentais, Av. Colombo, 5790, Bloco G-90, NUPELIA, Zona 07, 87020-900 Maringá, PR, Brasil

2. Universidade Federal de Goiás, Laboratório de Gerenciamento de Recursos Hídricos e Meio Ambiente (LAMARH), Departamento de Biologia Geral, Campus II, Caixa Postal 131, 74001-970 Goiânia, GO, Brasil

3. Universidade de Brasília, Instituto de Ciências Biológicas, Departamento de Botânica. Campus Universitário Darcy Ribeiro, Asa Norte, 70919-910 Brasilia, DF, Brasil

4. Autor para correspondência: dunck.barbara@gmail.com 
gênero foram acrescentados três novos táxons por Lange-Bertalot \& Metzeltin (2009), quatro novas espécies foram propostas por Wetzel et al. (2010) e uma espécie por Metzeltin \& Tremarin (2011).

No Brasil, estudos de Eunotia e Actinella presentes em águas continentais podem ser destacados: Metzeltin \& Tremarin (2011) descreveram uma nova espécie de Eunotia presente no perifíton aderido a Sphagnum L. na região sudeste; Bicca et al. (2011) avaliaram a família Eunotiaceae de ambientes lacustres na planície costeira do país; Wetzel et al. (2010) analisaram aspectos taxonômicos e biogeográficos do complexo das Eunotia asterionelloides da região amazônica; Faria et al. (2010) inventariaram diatomáceas perifíticas da represa Itaqui no Estado do Paraná, incluindo a ordem Eunotiales; Melo et al. (2010) inventariaram o gênero Actinella presente no plâncton de um lago de água escura da região amazônica; Bicca \& Torgan (2009) avaliaram novos registros de Eunotia para o Estado do Rio Grande do Sul de amostras fitoplanctônicas e perifíticas; Tremarin et al. (2008) descreveram 40 táxons para o gênero Eunotia presentes em ambiente lótico provenientes de amostras fitoplanctônicas e perifíticas, e apresentaram uma importante revisão dos principais trabalhos relacionados para o gênero Eunotia para a América do Sul e Estado do Paraná; Ferrari et al. (2007) analisaram as espécies da família Eunotiaceae presentes em igarapés da região amazônica; Bicudo et al. (1999) inventariaram as espécies da ordem Eunotiales presentes no Parque Estadual das Fontes do Ipiranga em São Paulo; Bicudo et al. (1995) avaliaram os táxons da família Eunotiaceae presentes na região do Pantanal em Poconé; e Rodrigues \& Moreira-Filho (1990) inventariaram as espécies da família Eunotiaceae do Rio Tubarão em Santa Catarina.

Para o Estado de Goiás apenas o trabalho de Souza \& Moreira-Filho (1999) avaliaram espécies da família Eunotiaceae apresentando detalhamentos taxonômicos para o grupo. Dado a escassez de estudos taxonômicos desta família para a região Centro-Oeste do Brasil, o presente trabalho teve como objetivo inventariar os táxons da família Eunotiaceae presentes no plâncton do Sistema Lago dos Tigres durante o período de águas baixas de 2004 .

\section{Material e métodos}

O Sistema Lago dos Tigres (Britânia,GO, $15^{\circ} 36^{\prime} 33,5^{\prime \prime}-15^{\circ} 22^{\prime} 8,7^{\prime \prime S}$ e $\left.51^{\circ} 17^{\prime} 56,3^{\prime \prime}-51^{\circ} 16^{\prime} 91^{\prime \prime} \mathrm{W}\right)$ é um vale bloqueado, composto por regiões lóticas, representado pelos rios Água Limpa e Vermelho, e de regiões lênticas no Lago dos Tigres. Esses tipos de lagos formados por vales bloqueados são formados por deposição de sedimentos que podem obstruir os tributários durante o enchimento do curso principal (Kalff 2002).

O estudo foi desenvolvido em 11 estações amostrais (figura 1), sendo três em zona lótica e oito na zona lacustre (tabela 1). Maiores descrições dos pontos amostrais, características morfométricas, variáveis limnológicas e climatológicas deste estudo encontram-se em Nabout \& Nogueira (2007).

Amostras de fitoplâncton foram coletadas na subsuperfície uma vez ao mês durante o período de seca (junho a setembro-2004) e início do período de chuva (outubro a novembro-2004), através de processos seletivos (rede de plâncton $25 \mu \mathrm{m}$ ), totalizando 66 amostras. Tais amostras foram fixadas com solução Transeau para estudos qualitativos (Bicudo \& Menezes 2006) e posteriormente depositadas no Herbário da UFG (números UFG29864 a UFG29939).

O material planctônico foi oxidado e limpo pela técnica de Simonsen (1974) modificada por Moreira-Filho \& Valente-Moreira (1981) e preparado em lâminas permanentes com resina Naphrax. As lâminas foram analisadas em microscópio óptico Zeiss Axioscop 40. Para a análise dos espécimes em microscopia eletrônica de varredura (MEV), o material foi preparado de acordo com Ferrario et al. (1995). As alíquotas de material oxidado foram depositadas em lamínulas e desidratadas com auxílio de luminária. Posteriormente, as lamínulas foram coladas em suportes metálicos com tinta carbono, metalizadas com ouro para observação em microscópio eletrônico (Jeol modelo JSM 6610). O sistema de classificação utilizado foi o proposto por Round et al. (1990) complementado por Medlin \& Kaczmarska (2004) e a identificação em literatura recente e atualizada.

\section{Resultados e Discussão}

No sistema Lago dos Tigres foram identificados 22 táxons, sendo uma espécie do gênero Actinella e 21 táxons infragenéricos de Eunotia. Apresenta-se a seguir a chave geral de identificação, as espécies identificadas, seguidas pela descrição, comentários, distribuição geográfica para o Centro-Oeste (baseada em artigos e capítulos de livros publicados) e ilustrações do material examinado. 


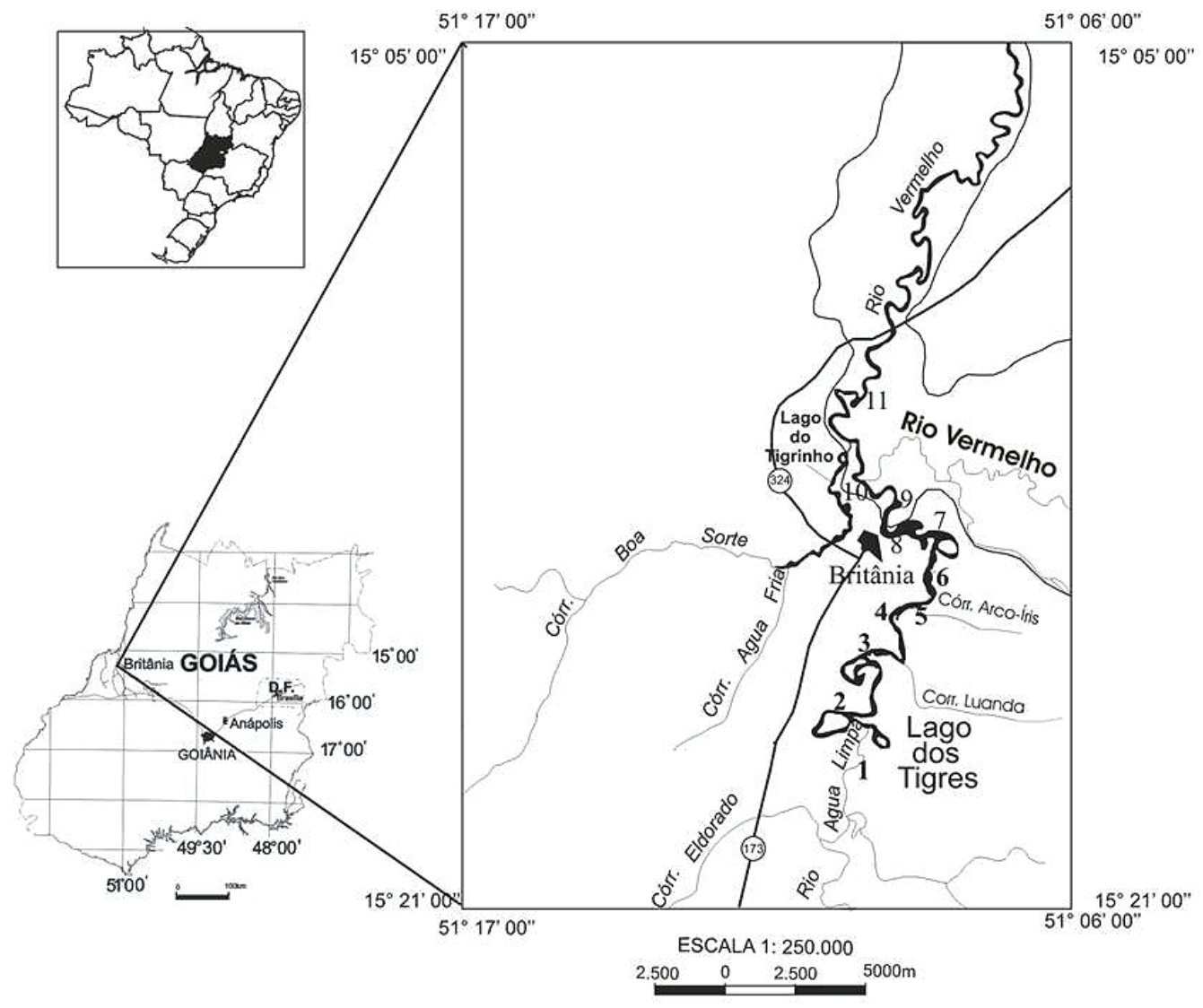

Figura 1- Sistema Lago dos Tigres, Britânia, GO, Brasil. Estações amostrais: de 1 a 11.

Figure 1- Sistema Lago dos Tigres, Britânia, Goiás State, Brasil. Sampling stations: 1 to 11.

Chave de identificação geral

1. Valvas heteropolares Actinella guianensis

1. Valvas isopolares

2. Presença de ondulações na margem dorsal da valva

3. Valvas com uma ondulação Eunotia rabenhorstii var. monodon

3. Valvas com mais de uma ondulação

4. Valvas com apenas duas ondulações

5. Valvas com ou sem intumescência na região mediana Eunotia didyma

5. Valvas sem intumescência

6. Valvas sem constricção na margem ventral Eunotia tropico-arcus

6. Valvas com leve ou forte constricção na margem ventral

7. Valvas com forte constricção na margem ventral Eunotia papilio

7. Valvas com discreta constricção na margem ventral Eunotia pileus

4. Valvas com duas ou mais ondulações

8. Valvas com duas ondulações com formato de corcovas

9. Apenas duas corcovas, extremidades rostradas Eunotia diodonopsis

9. Duas ou mais corcovas, extremidades arredondadas Eunotia camelus

8. Valvas com duas ou mais ondulações sem formato de corcovas

10. Valvas com duas ondulações Eunotia diodon 10. Valvas com mais de duas ondulações 
11. Valvas com três ondulações Eunotia trigibba

11. Valvas com quatro ondulações Eunotia zygodon

2. Ausência de ondulações na margem dorsal da valva

12. Extremidades mais largas que a largura mediana da valva

13. Valvas com uma discreta protuberância na região mediana da margem ventral ou margem dorsal

Eunotia formica

13. Valvas sem protuberância na região mediana nas margens

14. Valvas acima de $190 \mu \mathrm{m}$ de eixo apical Eunotiaflexuosa

14. Valvas até $155 \mu \mathrm{m}$ de eixo apical Eunotia rabenhorstiana var. elongata

12. Extremidades mais estreitas que a largura mediana da valva

15. Extremidades destacadas do corpo valvar Eunotia longicollis

15. Extremidades não destacadas ou levemente destacadas do corpo valvar

16. Extremidades não destacadas

17. Extremidades defletidas

18. Extremidades defletidas ventralmente

Eunotia deficiens

18. Extremidades defletidas dorsalmente Eunotia naegelii

17. Extremidades não defletidas

19. Eixo apical até $15 \mu \mathrm{m}$ Eunotia intermedia 19. Eixo apical superior a $29 \mu \mathrm{m}$ Eunotia sudetica

16. Extremidades levemente destacadas

20. Margem ventral quase reta Eunotia asterionelloides

20. Margem ventral convexa a levemente convexa

21. Eixo apical com até $70 \mu \mathrm{m}$ Eunotia monodon

21. Eixo apical superior a $90 \mu \mathrm{m}$ Eunotia major

Bacillariophytina Medlin \& Kaczmarska 2004

Bacillariophyceae Haeckel 1878 emend. Medlin

\& Kaczmarska 2004

Eunotiophycidae D. G. Mann in Round et al. 1990

Eunotiales Silva 1962

Eunotiaceae Kützing 1844

Actinella Lewis 1864

Eunotia Ehrenberg 1837

Actinella guianensis Grunow, Syn. Diat. Belgique: pl. XXXV: fig. 17. 1881.

Figuras 2-3

Valvas levemente curvadas, margem dorsal levemente convexa e margem ventral levemente côncava. Extremidade apical dilatada espatulada, ápice arredondado e extremidade basal cuneada-rostrada. Estrias transapicais paralelas em toda extensão da valva e levemente convergentes nas extremidades. Presença de pequenos espinhos nas margens da valva. Eixo apical 65-70 $\mu \mathrm{m}$, eixo transapical 5-9 $\mu \mathrm{m}$, 12 estrias em $10 \mu \mathrm{m}$. Espécimes presentes em ambiente lêntico.

Os espécimes inventariados representados pelas figuras 3 e 4 assemelham-se ao ilustrado por Hustedt em Schmidt et al. (1874-1959) para esta espécie quanto ao formato valvar, ápices dilatados espatulados e padrão de estriação. Diferem das espécies presentes em ambiente lêntico da região amazônica do Brasil determinadas por Melo et al. (2010), que apresentam maiores valores de eixo apical (112-121,4 $\mu \mathrm{m})$ e extremidade apical dilatada, cuneada-apiculada e extremidade basal cuneada.

Distribuição geográfica para a região Centro-Oeste: Planaltina (Souza \& Moreira-Filho 1999); Poconé (Bicudo et al. 1995).

Material examinado: BRASIL. GoiÁs: Britânia, Lago dos Tigres, Estação 4, plâncton, 4-VI-2004, Nogueira et al. 877 (UFG29866).

Eunotia Ehrenberg 1837

Eunotia asterionelloides Hustedt, Berichten der Deutschen Botanischen Gesellschaft, v.65, p.138, Pl. V figs.18-19. 1952.

Figuras 4-5, 49-51

Valvas levemente arqueadas, com margem dorsal fracamente convexa e ventral quase reta. Extremidades arredondadas levemente capitadas. Estrias transapicais paralelas em toda a extensão da valva. Eixo apical: 15-35 $\mu \mathrm{m}$; eixo transapical: 3-4 $\mu \mathrm{m}$; $16-19$ estrias em $10 \mu \mathrm{m}$. 
Tabela 1 - Localização dos pontos de amostragem no Sistema Lago dos Tigres, Britânica, GO, Brasil.

Table 1- Location of sampling stations at Sistema Lago dos Tigres, Britânia, Goiás State, Brazil.

\begin{tabular}{llll}
\hline Estação & Localidade & Latitude & Longitude \\
\hline 1 & Rio Água Limpa - Faz. Água Limpa (lótico) & $15^{\circ} 18^{\prime} 58^{\prime \prime} \mathrm{S}$ & $51^{\circ} 09^{\prime} 56^{\prime \prime} \mathrm{W}$ \\
2 & Baia Ilha das Perdidas (lêntico) & $15^{\circ} 17^{\prime} 57^{\prime \prime} \mathrm{S}$ & $51^{\circ} 10^{\prime} 07^{\prime \prime} \mathrm{W}$ \\
3 & Foz do Córrego Luanda - Faz. Santo Antônio (lêntico) & $15^{\circ} 16^{\prime} 47^{\prime \prime} \mathrm{S}$ & $51^{\circ} 09^{\prime} 44^{\prime \prime} \mathrm{W}$ \\
4 & Retiro da Faz. Santo Antônio (lêntico) & $15^{\circ} 16^{\prime} 17^{\prime \prime} \mathrm{S}$ & $51^{\circ} 09^{\prime} 10^{\prime \prime} \mathrm{W}$ \\
5 & Foz do Córrego Arco - Íris (lêntico) & $15^{\circ} 15^{\prime} 46^{\prime \prime} \mathrm{S}$ & $51^{\circ} 08^{\prime} 41^{\prime \prime} \mathrm{W}$ \\
6 & Ancoradouro das Balsas (lêntico) & $15^{\circ} 15^{\prime} 12^{\prime \prime} \mathrm{S}$ & $51^{\circ} 08^{\prime} 33^{\prime \prime} \mathrm{W}$ \\
7 & Faz. Lago dos Tigres (lêntico) & $15^{\circ} 14^{\prime} 17^{\prime \prime} \mathrm{S}$ & $51^{\circ} 08^{\prime} 59^{\prime \prime} \mathrm{W}$ \\
8 & Cidade de Britânia em frente ao monumento do Cristo (lêntico) & $15^{\circ} 14^{\prime} 09^{\prime \prime} \mathrm{S}$ & $51^{\circ} 09^{\prime} 27^{\prime \prime} \mathrm{W}$ \\
9 & Saída do Lago dos Tigres - Faz. Lago dos Tigres (lêntico) & $15^{\circ} 13^{\prime} 43^{\prime \prime} \mathrm{S}$ & $51^{\circ} 09^{\prime} 14^{\prime \prime} \mathrm{W}$ \\
10 & Foz do Lago dos Tigres no Rio Vermelho (lótico) & $15^{\circ} 13^{\prime} 18^{\prime \prime} \mathrm{S}$ & $51^{\circ} 10^{\prime} 06^{\prime \prime} \mathrm{W}$ \\
11 & Desvio Rio Vermelho (lótico) & $15^{\circ} 11^{\prime} 47^{\prime \prime} \mathrm{S}$ & $51^{\circ} 09^{\prime} 57^{\prime \prime} \mathrm{W}$ \\
\hline
\end{tabular}

Espécimes planctônicas que podem formar colônias em forma de estrela ou em zig-zag, e diferem de Eunotia loboi Wetzel \& Ector pela menor curvatura da valva e extremidades capitadas levemente voltadas para margem ventral (Wetzel et al. 2010).

Distribuição para a região Centro-Oeste: Goânia (Brandão \& Kravchenko 1997).

Material examinado: BRASIL. GoIÁs: Britânia, Lago dos Tigres, Estação 3, plâncton, 16-X-2004, Nogueira et al. 1025 (UFG29920); Lago dos Tigres, Estação 6, plâncton, 19-VIII-2004, Nogueira et al. 948 (UFG29898); Lago dos Tigres, Estação 4, plâncton, 4-VI-2004, Nogueira et al. 877 (UFG29866); Lago dos Tigres, Estação 6, plâncton, 16-X-2004, Nogueira et al. 1028 (UFG29923); Lago dos Tigres, Estação 5, plâncton, 13-VII-2004, Nogueira et al. 903 (UFG29886); Lago dos Tigres, Estação 6, plâncton, 9-IX-2004, Nogueira et al. 990 (UFG29912); Rio Vermelho, Estação 10, plâncton, 5-VI-2004, Nogueira et al. 880 (UFG29869); Rio Vermelho, Estação 10, plâncton, 14-VII-2004, Nogueira et al. 906 (UFG29889); Rio Vermelho, Estação 11, plâncton, 5-VI-2004, Nogueira et al. 881 (UFG29870); Rio Vermelho, Estação 11, plâncton, 5-VI-2004, Nogueira et al. 882 (UFG29871); Rio Água Limpa, Estação 1, plâncton, 14-VII-2004, Nogueira et al. 908 (UFG29891).

Eunotia camelus Ehrenberg, Phys. Abh. Akad. Wiss. Berlin, p. 413, pl.II, fig. I.1. 1841.

Figuras 10-11, 45-47
Valvas arqueadas, margem dorsal ondulada formando duas, quatro ou oito corcovas achatadas, margem ventral côncava, extremidades arredondadas e levemente defletidas dorsalmente. Estrias transapicais paralelas na região mediana e convergentes na extremidade. Nódulos terminais conspícuos próximos das extremidades. Eixo apical: 34-52 $\mu \mathrm{m}$; eixo transapical: 4-8 $\mu \mathrm{m}$; 10-12 a estrias em $10 \mu \mathrm{m}$.

Estes táxons podem apresentar morfotipos com duas, quatro ou oito corcovas na margem dorsal da valva (Schmidt et al. 1874-1959). Bicca et al. (2011) registraram diferentes morfotipos (E. camelus var. camelus e E. camelus var. denticulata) em mesmo ambiente, e por esse fato, descartam que as diferenças morfológicas encontradas entre os morfotipos são uma resposta à condições ambientais distintas.

Distribuição para a região Centro-Oeste: Planaltina (Souza \& Moreira-Filho1999), Goiânia (Brandão \& Kravchenko 1997, Macedo-Saidah et al. 1987), Poconé (Bicudo et al. 1995), Itajá (Contin \& Oliveira 1993).

Material examinado: BRASIL. GoIÁs: Rio Água Limpa, Estação 1, plâncton, 9-IX-2004, Nogueira et al. 985 (UFG29907); Lago dos Tigres, Estação 2, plâncton, 13-VII-2004, Nogueira et al. 898 (UFG29880); Lago dos Tigres, Estação 3, plâncton, 13-VII-2004, Nogueira et al. 899 (UFG29881); Lago dos Tigres, Estação 7, plâncton, 4-VI-2004, Nogueira et al. 878 (UFG29867); Lago dos Tigres, Estação 8, plâncton, 13-VII-2004, Nogueira et al. 904 (UFG29887); Lago dos Tigres, Estação 4, plâncton, 4-VI-2004, 
Nogueira et al. 877 (UFG29866); Rio Vermelho, Estação 10, plâncton, 5-VI-2004, Nogueira et al. 880 (UFG29869); Rio Vermelho, Estação 11, plâncton, 5-VI-2004, Nogueira et al. 881 (UFG29870); Rio Água Limpa, Estação 1, plâncton, 14-VII-2004, Nogueira et al. 908 (UFG29891); Rio Vermelho, Estação 11, plâncton, 17-X-2004, Nogueira et al. 1033 (UFG29928).

Eunotia deficiens Metzeltin, Langue-Bertalot \& Garcia-Rodriguez, Iconogr. Diatomol. 15: 48, pl. 22: fig. 5. 2005.

Figuras 27-28

Valvas com margem dorsal levemente convexa e margem ventral reta. Extremidades atenuoarredondadas, defletidas ventralmente. Estrias transapicais paralelas em toda extensão da valva e fortemente convergente nas extremidades. Nódulos terminais conspícuos afastados das extremidades próximos da margem ventral. Eixo apical: 51-90 $\mu \mathrm{m}$; eixo transapical: 9-12 $\mu \mathrm{m} ; 6$ a 9 estrias em $10 \mu \mathrm{m}$.

Esta espécie assemelha-se a Eunotia luna var. aequalis f. major Hustedt, no entanto E. luna var. aequalis f. major além de apresentar maiores valores de eixo apical e transapical e menor número de estrias em $10 \mu \mathrm{m}$, apresenta extremidades mais alongadas e voltadas ventralmente, diferentemente de E. deficiens (Frenguelli 1933, pág. 491, prancha VIII, fig. 1).

Distribuição para a região Centro-Oeste Federal: citação pioneira.

Material examinado: BRASIL. GoIÁs: Rio Água Limpa, Estação 1, plâncton, 9-IX-2004, Nogueira et al. 985 (UFG29907); Lago dos Tigres, Estação 6, plâncton, 9-IX-2004, Nogueira et al. 989 (UFG29911); Lago dos Tigres, Estação 3, plâncton, 16-X-2004, Nogueira et al. 1025 (UFG29920).

Eunotia didyma Hustedt ex Zimmerman, Broteria, Serie Botanica, vol.13 (1-2) pp. 51-52, pl. 5, figs. 4-6, pl. 6, fig. 13. 1915.

Figuras 13-17

Valvas levemente arqueadas com duas ondulações pronunciadas ou pouco pronunciadas na margem dorsal, margem dorsal levemente convexa, margem ventral levemente côncava. Presença de intumescência arredondada região mediana em ambas as margens em alguns morfotipos. Extremidades cuneadas pouco a alongadas. Estrias transapicais paralelas na região mediana, levemente a fortemente radiadas nas extremidades. Nódulos terminais conspícuos nas extremidades. Eixo apical: 38-100 $\mu \mathrm{m}$; eixo transapical: $12-15 \mu \mathrm{m} ; 8-14$ estrias em $10 \mu \mathrm{m}$.

O complexo Eunotia didyma apresenta dois grupos distintos de variedades, um com valvas mais alongadas e presença de dilatação na região central e outro com valvas bilobadas ou com duas ondulações na margem dorsal da valva (Patrick 1940). Os espécimes estudados assemelham-se ao ilustrado por Hustedt em A. Schmidt (1913, pr. 285, figs. 19-22), Frenguelli (1941, pr. V, fig. 5) e Patrick (1940, pr. 8, fig. 21) para a variedade típica. Os morfotipos com dilatação na região central assemelham-se a $E$. formica Ehrenberg, no entanto em E. formica os ápices são dilatados e menos alongados que nestes morfotipos, também descritos como E. didyma var. claviculata (Patrick 1940). Esses morfotipos assemelham-se ao ilustrado por Hustedt em A. Schmidt (1913, pr. 285, fig. 16), por Compère (1975, pr. 2, fig. 59) e Metzeltin \& Lange-Bertalot (1998, pág. 551, pr. 160, fig. 12).

Distribuição para Goiás e Distrito Federal: Lagoa Bonita (Souza \& Moreira-Filho 1999), Poconé (Bicudo et al. 1995), Itajá (Contin \& Oliveira 1993).

Material examinado: BRASIL. GoIÁs: Britânia, Rio Vermelho, Estação 11, plâncton, 5-VI-2004, Nogueira et al. 881 (UFG29870), Lago dos Tigres, Estação 3, plâncton, 9-IX-2004, Nogueira et al. 987 (UFG29909); Lago dos Tigres, Estação 2, plâncton, 13-VII-2004, Nogueira et al. 898 (UFG29880); Lago dos Tigres, Estação 7, plâncton, 4-VI-2004, Nogueira et al. 878 (UFG29867); Lago dos Tigres, Estação 4, plâncton, 4-VI-2004, Nogueira et al. 877 (UFG29866); Rio Vermelho, Estação 11, plâncton, 17-X-2004, Nogueira et al. 1033 (UFG29928). Lago dos Tigres, Estação 7, plâncton, 13-VII-2004, Nogueira et al. 903 (UFG29886); Lago dos Tigres, Estação 8, plâncton, 13-VII-2004, Nogueira et al. 904 (UFG29887); Rio Água Limpa, Estação 1, plâncton, 09-IX-2004, Nogueira et al. 985 (UFG29907).

Eunotia diodon Ehrenberg, Ber. K. Akad. Wiss. Berlin 1837: 45.1837.

Figuras 8-9, 48

Valvas arqueadas, margem dorsal com duas ondulações, margem ventral levemente côncava com a região mediana levemente pronunciada arredondada, extremidades arredondadas e levemente defletidas dorsalmente. Estrias transapicais paralelas em toda a extensão da valva. Nódulos terminais conspícuos 
próximos à margem ventral. Eixo apical: $25-28 \mu \mathrm{m}$; Eixo transapical: 5-7 $\mu \mathrm{m}$; 10-12 estrias em $10 \mu \mathrm{m}$.

Este táxon assemelha-se a Eunotia camelus Ehrenberg quanto à morfologia da valva, diferem porém por ter menores valores de eixo apical, apenas duas ondulações, estrias paralelas em toda a extensão da valva e leve concavidade na margem ventral (Patrick \& Reimer 1966).

Distribuição para a região Centro-Oeste: Goiânia (Brandão \& Kravchencko 1997)

Material examinado: BRASIL. GoIÁs: Britânia, Lago dos Tigres, Estação 4, plâncton, 4-VI-2004, Nogueira et al. 877 (UFG29866); Rio Água Limpa, Estação 1, plâncton, 14-VII-2004, Nogueira et al. 908 (UFG29891).

Eunotia diodonopsis Metzeltin, Langue-Bertalot \& Garcia-Rodriguez, Iconogr. Diatomol. 15: 50, pl. 25: figs. 1-11. 2005.

Figuras 20-21

Valvas arqueadas, margem dorsal ondulada formando corcovas achatadas, margem ventral côncava, extremidades rostrado-truncadas. Estrias transapicais paralelas na região mediana e levemente convergentes na extremidade. Nódulos terminais conspícuos. Eixo apical: $38-41 \mu \mathrm{m}$; eixo transapical: 9-10 $\mu \mathrm{m}$; 11 a 12 estrias em $10 \mu \mathrm{m}$.

Eunotia diodonopsis assemelha-se a E. praerupta var. bidens, no entanto difere quanto a curvatura da margem ventral, que em E. diodonopsis é mais acentuada, padrão de estriação, ondulações truncadas e ápices, que em E. praerupta var. bidens são levemente defletidos dorsalmente (Frenguelli 1941, pr. V, fig. 9; Patrick \& Reimer 1966, pag. 226, pr. 10, fig. 12). A morfologia valvar de E. diodonopsis ainda é semelhante a $E$. eruca var. siberica Cleve-Euler ilustrado por Zalocar de Domitrovic \& Maidana para espécies do Rio Paraná na Argentina (1997, pag. 55, pr. VI, fig. 11), no entanto a posição dos nódulos terminais difere entre as espécies.

Distribuição para a região Centro-Oeste: citação pioneira.

Material examinado: BRASIL. GoIÁs: Britânia, Rio Vermelho, Estação 11, plâncton, 5-VI-2004, Nogueira et al. 881 (UFG29870) Lago dos Tigres, Estação 2, plâncton, 13-VII-2004, Nogueira et al. 898 (UFG29880).
Eunotia flexuosa (Brébisson in Kützing) Kützing, Spec. Algar., p. 6, 1849.

Figuras 43-44

Valvas lineares levemente paralelas, margem dorsal levemente convexa e margem ventral levemente côncava. Extremidades arredondadas espatuladas diferenciadas do corpo valvar. Estrias transapicais paralelas na região mediana e levemente convergentes na extremidade. Nódulos terminais conspícuos nas extremidades. Eixo apical: 190-195 $\mu \mathrm{m}$; eixo transapical: 6-7 $\mu \mathrm{m}$; 17 estrias em $10 \mu \mathrm{m}$.

A presença dos nódulos terminais conspícuos nas extremidades são características marcantes deste táxon, como ilustrado por Metzeltin \& Lange-Bertalot (1998, pág. 511, pr. 140, figs. 8-18).

Distribuição para a região Centro-Oeste: Formosa, São João da Aliança (Souza \& Oliveira 2007), Lagoa Bonita (Souza \& Moreira-Filho 1999), Poconé (Bicudo et al. 1995); Goiânia (Macedo-Saidah et al. 1987; Brandão \& Kravchencko 1997).

Material examinado: BRASIL. GoIÁs: Britânia, Rio Água Limpa, Estação 1, plâncton, 9-IX-2004, Nogueira et al. 985 (UFG29907); Lago dos Tigres, Estação 7, plâncton, 4-VI-2004, Nogueira et al. 878 (UFG29867); Lago dos Tigres, Estação 4, plâncton, 4-VI-2004, Nogueira et al. 877 (UFG29866).

Eunotia formica Ehrenberg, Ber. K. Akad. Wiss. Berlin 1841: 414. 1843.

Figuras 37, 42

Valvas com margem dorsal levemente convexa e margem ventral reta a levemente côncava com discreta protuberância na região mediana de ambas as margens ou apenas em uma. Extremidades arredondadocuneadas, levemente capitada diferenciada ou não do corpo valvar. Estrias transapicais paralelas ao longo da valva. Nódulos terminais conspícuos nas extremidades próximos da margem ventral. Eixo apical: $40-180 \mu \mathrm{m}$; eixo transapical: $8-12 \mu \mathrm{m}$; 10 a 14 estrias em $10 \mu \mathrm{m}$. Características valvares dos táxons estudados assemelham-se ao descrito para espécie por Frenguelli (1941), no entanto os espécimes avaliados apresentaram maior número de estrias $(\mathrm{em} 10 \mu \mathrm{m})$ que o descrito para a espécie (5-6 estrias). Exemplares semelhantes foram ilustrados pelo mesmo autor (pr. VIII, figs. 5-6) e por Metzeltin \& Lange-Bertalot (1998, pág. 535, pr. 152, figs. 11-12).

Distribuição para a região Centro-Oeste: Poconé (Bicudo et al. 1995). 
Material examinado: BRASIL. GoiÁs: Britânia, Rio Água Limpa, Estação 1, plâncton, 9-IX-2004, Nogueira et al. 985 (UFG29907); Lago dos Tigres, Estação 2, plâncton, 13-VII-2004, Nogueira et al. 898 (UFG29880); Rio Vermelho, Estação 10, plâncton, 14-VII-2004, Nogueira et al. 906 (UFG29889); Rio Vermelho, Estação 11, plâncton, 14-VII-2004, Nogueira et al. 907 (UFG29890).

Eunotia intermedia (Krasske ex Hustedt) Nörpel \& Lange-Bertalot, In Lange-Bertalot, Biblioth. Diatomol. 27: 32. 1993.

Figuras 32-33

Valvas com margem dorsal convexa, margem ventral reta. Extremidade arredondada, nódulo terminal ventral conspícuo próximo da extremidade. Estrias delgadas transapicais na região mediana da valva e levemente radiadas na extremidade. Eixo apical: 14-30 $\mu \mathrm{m}$; eixo transapical: 4-6 $\mu \mathrm{m}$; 9-10 estrias em $10 \mu \mathrm{m}$.

Este táxon apresenta características diacríticas semelhantes a Eunotia intermedia (Krasske ex Hustedt) Nörpel \& Lange-Bertalot, porém alguns espécimes apresentaram-se com maior largura do eixo transapical e menor número de estrias em $10 \mu \mathrm{m}$. Eunotia intermedia apresenta morfologia valvar semelhante a $E$. faba. No entanto $E$. faba apresenta maiores proporções em relação a E. intermedia (Metzeltin \& Lange-Bertalot 1998), e maior número de estrias em $10 \mu \mathrm{m}$ (14-19) enquanto que em E. intermedia (10-20 estrias) Krammer \& LangeBertalot (1998). Dessa forma, no presente estudo optou-se por identificar os exemplares analisados como E. intermedia.

Distribuição para a região Centro-Oeste: citação pioneira.

Material examinado: BRASIL. GOIÁS: Britânia, Lago dos Tigres, Estação 3, plâncton, 13-VII-2004, Nogueira et al. 899 (UFG29881); Lago dos Tigres, Estação 7, plâncton, 16-X-2004, Nogueira et al. 1029 (UFG29924).

Eunotia longicollis Metzeltin \& Lange-Bertalot, In Lange-Bertalot, Iconographia Diatomologica 5: 66. pl.15, figs.6-8, 1998.

Figura 31

Valvas levemente arqueadas a arqueadas, margem dorsal convexa, margem ventral levemente côncava a quase reta. Extremidade arredondada-rostrada reta. Estrias transapicais paralelas em toda extensão da valva. Eixo apical: 50-69 $\mu \mathrm{m}$, eixo transapical: 7-8 $\mu \mathrm{m}, 10$ estrias em $10 \mu \mathrm{m}$.

Os espécimes estudados assemelham-se ao Eunotia longicollis Metzeltin \& Lange-Bertalot (1998) prancha 15 figs. 6-8, porém alguns indivíduos apresentaram menores dimensões dos eixos apicais que o proposto pela bibliografia.

Distribuição para a região Centro-Oeste: citação pioneira.

Material examinado: BRASIL. GoIÁs: Britânia, Lago dos Tigres, Estação 5, plâncton, 9-IX-2004, Nogueira et al. 989 (UFG29911); Rio Vermelho, Estação 11, plâncton, 5-VI-2004, Nogueira et al. 881 (UFG29870).

Eunotia major (Smith) Rabenhorst, Fl. Eur. Alg. Sect. 1, pg. 72. 1864.

Figuras 35-36, 52-53

Valvas levemente arqueadas, margem dorsal levemente convexa e margem ventral côncava, extremidades arredondadas levemente defletidas dorsalmente, estrias transapicais paralelas na região mediana da valva e levemente convergentes na extremidade valvar. Eixo apical: 90-108 $\mu \mathrm{m}$; eixo transapical: $8-9 \mu \mathrm{m}$; 8 a 9 estrias em $10 \mu \mathrm{m}$.

Os espécimes estudados apresentam menores valores que o registrado para espécie por Frenguelli (1941), no entanto padrão e número de estrias são semelhantes (pr. VII, fig. 7). Esta espécie apresenta características próximas a Eunotia monodon Ehrenberg pela curvatura e morfologia das valvas, porém diferem pela presença de extremidades cuneado-arredondadas e nódulos terminais conspícuos em E. monodon (Van Heurck 1880-1885). Os espécimes analisados assemelham-se ao ilustrado por Metzeltin et al. 2005 para material de Uruguai (pag. 279, pr. 17, figs. 4-5).

Distribuição para a região Centro-Oeste: Lagoa Bonita (Souza \& Moreira-Filho 1999), Goiânia (Macedo-Saidah et al. 1987).

Material examinado: BRASIL. GoIÁs: Britânia, Lago dos Tigres, Estação 7, plâncton, 4-VI-2004, Nogueira et al. 878 (UFG29867); Lago dos Tigres, Estação 4, plâncton, 4-VI-2004, Nogueira et al. 877 (UFG29866); Rio Água Limpa, Estação 1, plâncton, 16-X-2004, Nogueira et al. 1023 (UFG29918).

Eunotia monodon Ehrenberg, Ber. K. Akad. Wiss. Berlin 1841: 206. 1841.

Figura 34 
Valvas levemente arqueadas, margem dorsal convexa e margem ventral levemente côncava, extremidades cuneado-arredondadas. Estrias transapicais paralelas em toda a extensão da valva, rafe indistinta. Nódulos terminais conspícuos nas extremidades próximos da margem ventral. Eixo apical: $66-70 \mu \mathrm{m}$; eixo transapical: 9-10 $\mu \mathrm{m}$; 12-13 estrias em $10 \mu \mathrm{m}$.

O padrão de estriação e posição dos nódulos terminais dos espécimes estudados conferem ao ilustrado por Frenguelli (1941, fig. 17 pr. VII). Eunotia monodon apresentam formato valvar semelhante a Eunotia pseudoindica, porém as espécies diferem por E. pseudoindica apresentar margem valvar dorsal levemente convexa, extremidades cuneado-afiladas destacadas do corpo, estrias paralelas a radiadas em direção as extremidades e maior número de estrias em $10 \mu \mathrm{m}$ (Bicca et al. 2011).

Distribuição para a região Centro-Oeste: Itajá (Contin \& Oliveira 1993), Goiânia (Brandão \& Kravchenko 1997, Macedo-Saidah et al. 1987); Poconé (Bicudo et al. 1995).

Material examinado: BRASIL. Goiás: Britânia, Lago dos Tigres, Estação 2, plâncton, 13-VII-2004, Nogueira et al. 898 (UFG29880).

Eunotia naegelii Migula in Thomé, Fr. Deutschland, v.2, n.1, p. 203, 1907.

Figuras 40-41

Valvas arqueadas, margem dorsal convexa e ventral côncava, extremidades arredondadas levemente defletidas dorsalmente. Estrias transapicais delicadas e paralelas em toda a extensão da valva, rafe indistinta. Nódulos terminais conspícuos nas extremidades próximos da margem ventral. Eixo apical: $90-95 \mu \mathrm{m}$; eixo transapical: $3-5 \mu \mathrm{m}$; 25 estrias em $10 \mu \mathrm{m}$, razão comprimento/largura: 19.

Os espécimes analisados apresentaram maior número de estrias que o descrito para a espécie por Patrick \& Reimer (1966). Eunotia naegelii apresenta morfologia semelhante a E. bilunaris e a E. curvata, no entanto os ápices levemente defletidos dorsalmente de E. naegelii diferem de E. bilunaris, e em relação a proporção comprimento/largura, em E. curvata é 27:1, maior que em E. naegelii (Patrick \& Reimer 1966).

Distribuição para a região Centro-Oeste: Lagoa Bonita (Souza \& Moreira-Filho 1999).

Material examinado: BRASIL. GoIÁs: Britânia, Lago dos Tigres, Estação 7, plâncton, 4-VI-2004,
Nogueira et al. 878 (UFG29867); Lago dos Tigres, Estação 4, plâncton, 4-VI-2004, Nogueira et al. 877 (UFG29866); Lago dos Tigres, Estação 7, plâncton, 13-VII-2004, Nogueira et al. 903 (UFG29886); Lago dos Tigres, Estação 8, plâncton, 13-VII-2004, Nogueira et al. 904 (UFG29887).

Eunotia papilio (Ehrenberg) Grunow, Alg. Novara: 94. 1868.

Figuras 6-7

Valvas com margem dorsal convexa com duas ondulações e ventral fortemente côncava. Extremidades cuneadas defletidas dorsalmente. Estrias transapicais centrais paralelas e radiadas em direção às extremidades. Eixo apical: 30-32 $\mu \mathrm{m}$; eixo transapical: 7-11 $\mu \mathrm{m} ; 10$ estrias em $10 \mu \mathrm{m}$.

Os espécimes analisados assemelham-se ao ilustrado para a espécie em Reichardt (1995, pag. 45, pr. 2 , figs. 1 e 6 ) tanto no formato valvar como nas aréolas e o padrão de estriação.

Distribuição para a região Centro-Oeste: citação pioneira.

Material examinado: BRASIL. GoIÁs: Britânia, Lago dos Tigres, Estação 3, plâncton, 19-VIII-2004, Nogueira et al. 945 (UFG29895); Lago dos Tigres, Estação 7, plâncton, 4-VI-2004, Nogueira et al. 878 (UFG29867); Lago dos Tigres, Estação 2, plâncton, 13-VII-2004, Nogueira et al. 898 (UFG29880).

Eunotia pileus Ehrenberg, Ber. K. Akad. Wiss. Berlin, Physik. K1. 1841: 414. 1843

Figura 12

Valvas com margem dorsal convexa apresentando duas ondulações pouco pronunciadas, margem ventral levemente côncava. Extremidades pouco alongadas e cuneadas. Estrias transapicais paralelas, mais distantes na região mediana e levemente radiadas nas extremidades. Nódulos terminais inconspícuos. Eixo apical: 21-23 $\mu \mathrm{m}$; eixo transapical: 9-11 $\mu \mathrm{m}$; 9 estrias em $10 \mu \mathrm{m}$.

Os exemplares estudados assemelham-se a Eunotia didyma var. inflata, no entanto algumas características morfológicas diferem, como maior concavidade na margem ventral em E. didyma var. inflata, ápices mais alongados, menores valores de eixo transapical $(6-7 \mu \mathrm{m})$ e mais estrias em $10 \mu \mathrm{m}$ (15) (Patrick 1940) que E. pileus. Os espécimes analisados assemelham-se ao ilustrado para a espécie por Reichardt (1995, pag. 49, pr. 4, figs. 3, 10-11). 
Distribuição para a região Centro-Oeste: citação pioneira.

Material examinado: BRASIL. GoIÁs: Britânia, Rio Vermelho, Estação 11, plâncton, 17-X-2004, Nogueira et al. 1033 (UFG29928).

Eunotia rabenhorstiana (Grunow) Hustedt var. elongata (Patrick) Metzeltin \& Lange-Bertalot, In Lange-Bertalot, Iconographia Diatomologica 5: 76.pl. 64, fig. 1-13, pl. 65, fig. 17-19. 1998.

Figuras 38-39

Valvas levemente arqueadas quase paralelas, margem dorsal levemente convexa e margem ventral levemente côncava. Extremidade arredondadocuneada, nódulo terminal conspícuo nas extremidades próximo da margem ventral. Estrias transapicais paralelas na região mediana e levemente convergentes na extremidade. Eixo apical: 148-155 $\mu \mathrm{m}$, eixo transapical: $7-9 \mu \mathrm{m} ; 18$ estrias em $10 \mu \mathrm{m}$.

Espécimes analisados apresentaram características semelhantes ao descrito para Eunotia rabenhorstiana var. elongata; no entanto os espécimes apresentaram maior largura que o descrito para a variedade $(5-7 \mu \mathrm{m})$ (Patrick \& Reimer 1966). Esta espécie assemelha-se a E. transfuga Metzeltin \&Lange-Bertalot, no entanto difere por apresentar ápice mais estreito (Metzeltin \& Lange-Bertalot 1998).

Distribuição para a região Centro-Oeste: citação pioneira.

Material examinado: BRASIL. GoIÁs: Britânia, Lago dos Tigres, Estação 4, plâncton, 4-VI-2004, Nogueira et al. 877 (UFG29866); Lago dos Tigres, Estação 2, plâncton, 13-VII-2004, Nogueira et al. 898 (UFG29880); Lago dos Tigres, Estação 5, plâncton, 4-VI-2004, Nogueira et al. 878 (UFG29867); Lago dos Tigres, Estação 3, plâncton, 19-VIII-2004, Nogueira et al. 945 (UFG29895); Rio Água Limpa, Estação 1, plâncton, 9-VI-2004, Nogueira et al. 985 (UFG29907); Rio Vermelho, Estação 11, plâncton, 5-VI-2004, Nogueira et al. 881 (UFG29870).

Eunotia rabenhorstii var. monodon Grunow, Van Heurck, Syn. Diat. Belg. P1.XXXV: fig.12B. 1881. Figuras 18-19

Valvas com margem dorsal convexa com uma ondulação atenuo-arredondada na região mediana, margem ventral côncava, extremidades arredondadas. Estrias transapicais paralelas em toda a extensão da valva, rafe indistinta. Nódulos terminais conspícuos nas extremidades próximos da margem ventral.

Eixo apical: $23-25 \mu \mathrm{m}$; eixo transapical: 6-7 $\mu \mathrm{m}$; 12 a 14 estrias em $10 \mu \mathrm{m}$.

Todos os espécimes analisados apresentaram uma ondulação na margem dorsal, caracterizando a variedade monodon para a espécie determinada (Patrick \& Reimer 1966). Os espécimes analisados assemelham-se ao ilustrado por Patrick \& Reimer (1966) para a variedade (pag. 232, pr. 13, fig. 19).

Distribuição para a região Centro-Oeste: citação pioneira.

Material examinado: BRASIL. GoIÁs: Britânia, Rio Água Limpa, Estação 1, plâncton, 9-VI-2004, Nogueira et al. 985 (UFG29907); Rio Vermelho, Estação 11, plâncton, 5-VI-2004, Nogueira et al. 881 (UFG29870); Lago dos Tigres, Estação 2, plâncton, 13-VII-2004, Nogueira et al. 898 (UFG29880).

Eunotia sudetica O. Müller, Forschungsber. Biol. Stat. Plön. 6: 12, pl. 3, fig. 25-26. 1898.

Figuras 25-26

Valvas com margem dorsal convexa e margem ventral paralela reta. Extremidade arredondada, nódulos terminais conspícuos próximos das extremidades na margem ventral. Estrias transapicais paralelas em toda extensão da valva. Eixo apical: 29-32 $\mu \mathrm{m}$, eixo transapical: 5-9 $\mu \mathrm{m}, 9$ a 11 estrias em $10 \mu \mathrm{m}$.

Eunotia sudetica apresenta morfologia semelhante a $E$. incisa, porém em $E$. incisa o número de estrias em $10 \mu \mathrm{m}$ (13-17) é maior que em E. sudetica (8-13), e quanto aos ápices, que em E. sudetica são mais estreitos que o corpo valvar, e em $E$. incisa não são tão diferenciados (Patrick \& Reimer 1966).

Distribuição para a região Centro-Oeste: Formosa, São João da Aliança (Souza \& Oliveira 2007), Poconé (Bicudo et al. 1995), Itajá (Contin \& Oliveira 1993).

Material examinado: BRASIL. GoIÁs: Britânia, Rio Água Limpa, Estação 1, plâncton, 9-VI-2004, Nogueira et al. 985 (UFG29907); Lago dos Tigres, Estação 2, plâncton, 13-VII-2004, Nogueira et al. 898 (UFG29880); Lago dos Tigres, Estação 6, plâncton, 19-VIII-2004, Nogueira et al. 948 (UFG29898); Rio Vermelho, Estação 10, plâncton, 5-VI-2004, Nogueira et al. 880 (UFG29867); Lago dos Tigres, Estação 7, plâncton, 13-VII-2004, Nogueira et al. 903 (UFG29886). 

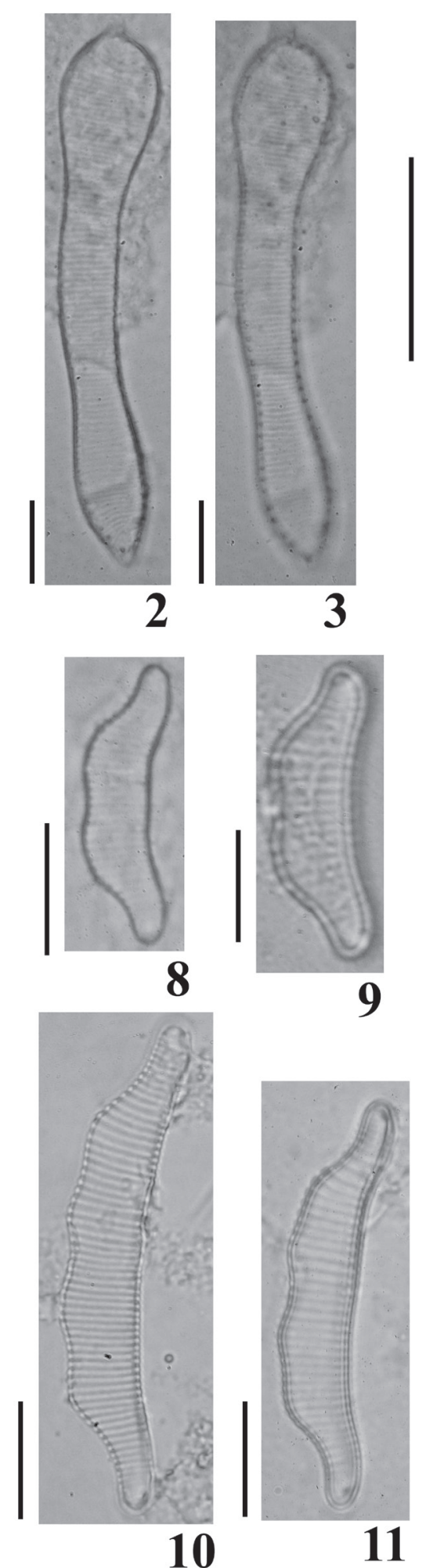
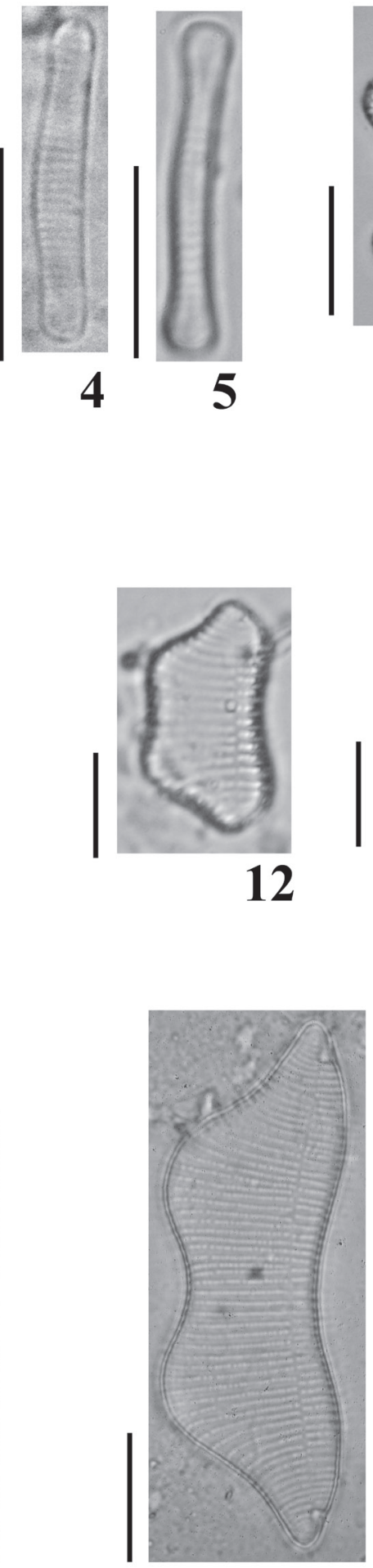

15
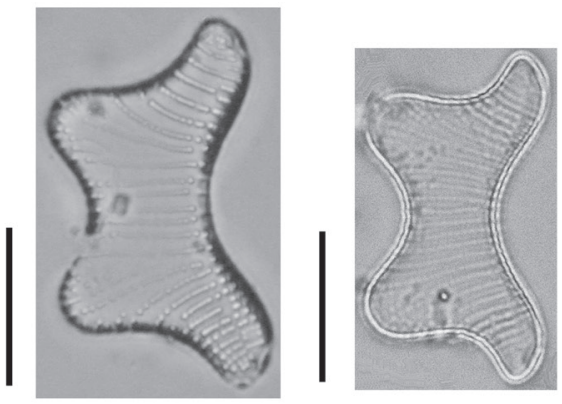

6
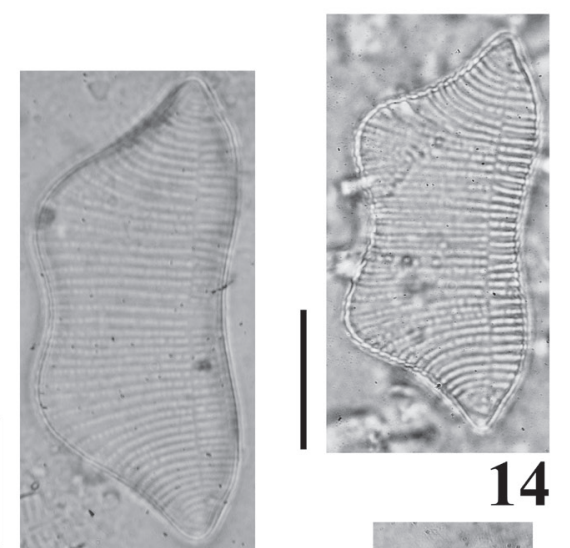

14
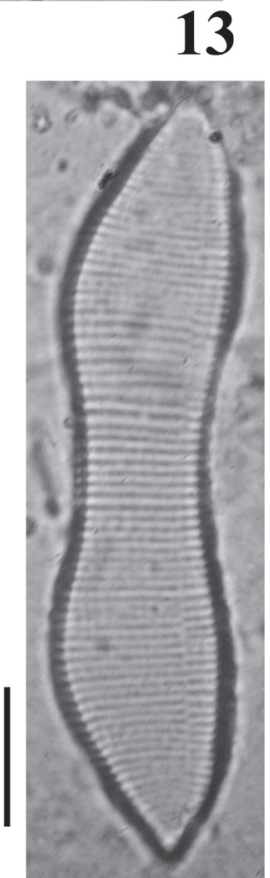

16

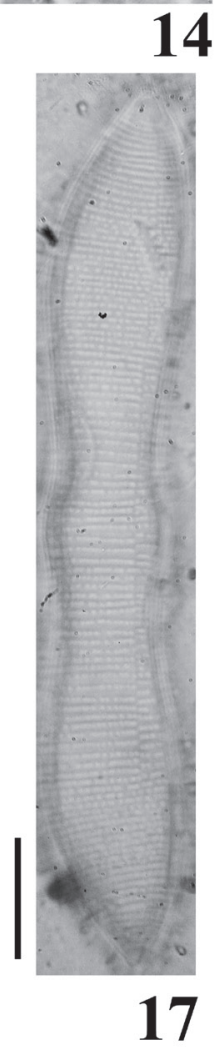

Figuras 2-17. Eunotiaceae Kützing (Bacillariophyceae) planctônicas do Sistema Lago dos Tigres, Britânia, GO, Brasil. 2-3. Actinella guianensis. 4-5. Eunotia asterionelloides. 6-7. Eunotia papilio. 8-9. Eunotia diodon.10-11. Eunotia camelus. 12. Eunotia pileus.13-17. Eunotia didyma. Barras de escala: $10 \mu \mathrm{m}$.

Figures 2-17. Planktonic Eunotiaceae Kützing (Bacillariophyceae) of Sistema Lago dos Tigres, Britânia, Goiás State, Brazil. 2-3. Actinella guianensis. 4-5. Eunotia asterionelloides. 6-7. Eunotia papilio. 8-9. Eunotia diodon.10-11. Eunotia camelus. 12. Eunotia pileus.13-17. Eunotia didyma. Scale bars: $10 \mu \mathrm{m}$. 

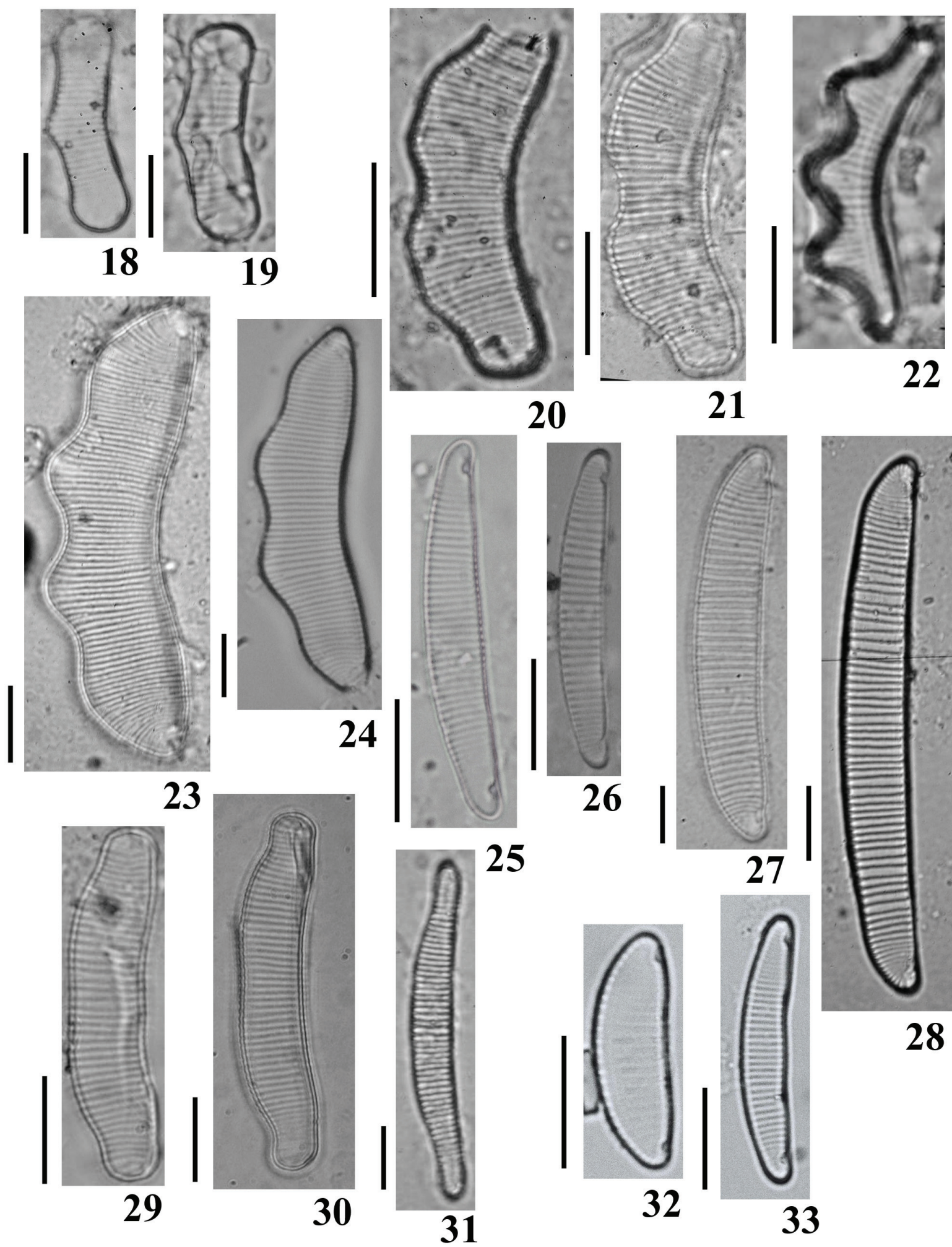

22

20

21
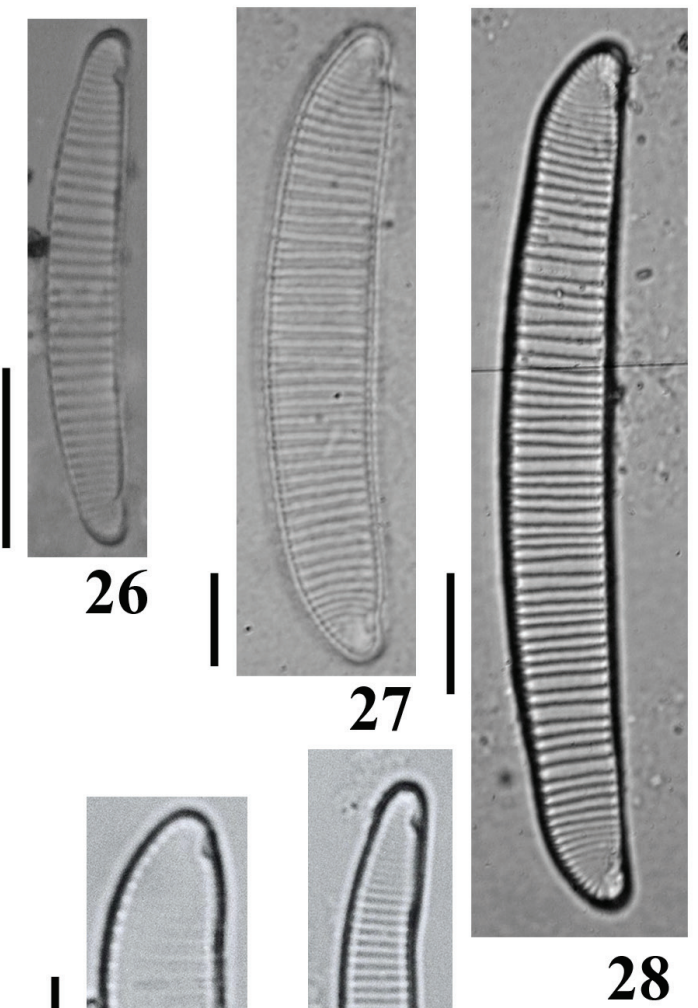

Figuras 18-33. Eunotiaceae Kützing (Bacillariophyceae) planctônicas do Sistema Lago dos Tigres, Britânia, GO, Brasil. 18-19. Eunotia rabennhorstii var. monodon. 20-21. Eunotia diodonopsis. 22. Eunotia trigibba. 23-24. Eunotia zygodon. 25-26. Eunotia sudetica. 27-28. Eunotia deficiens. 29-30. Eunotia tropico-arcus. 31. Eunotia longicollis. 32-33. Eunotia intermedia. Barras de escala: $10 \mu \mathrm{m}$.

Figures 18-33. Planktonic Eunotiaceae Kützing (Bacillariophyceae) of Sistema Lago dos Tigres, Britânia, Goiás State, Brazil. 18-19. Eunotia rabennhorstii var. monodon. 20-21. Eunotia diodonopsis. 22. Eunotia trigibba. 23-24. Eunotia zygodon. 25-26. Eunotia sudetica. 27-28. Eunotia deficiens. 29-30. Eunotia tropico-arcus. 31. Eunotia longicollis. 32-33. Eunotia intermedia. Scale bars: $10 \mu \mathrm{m}$. 

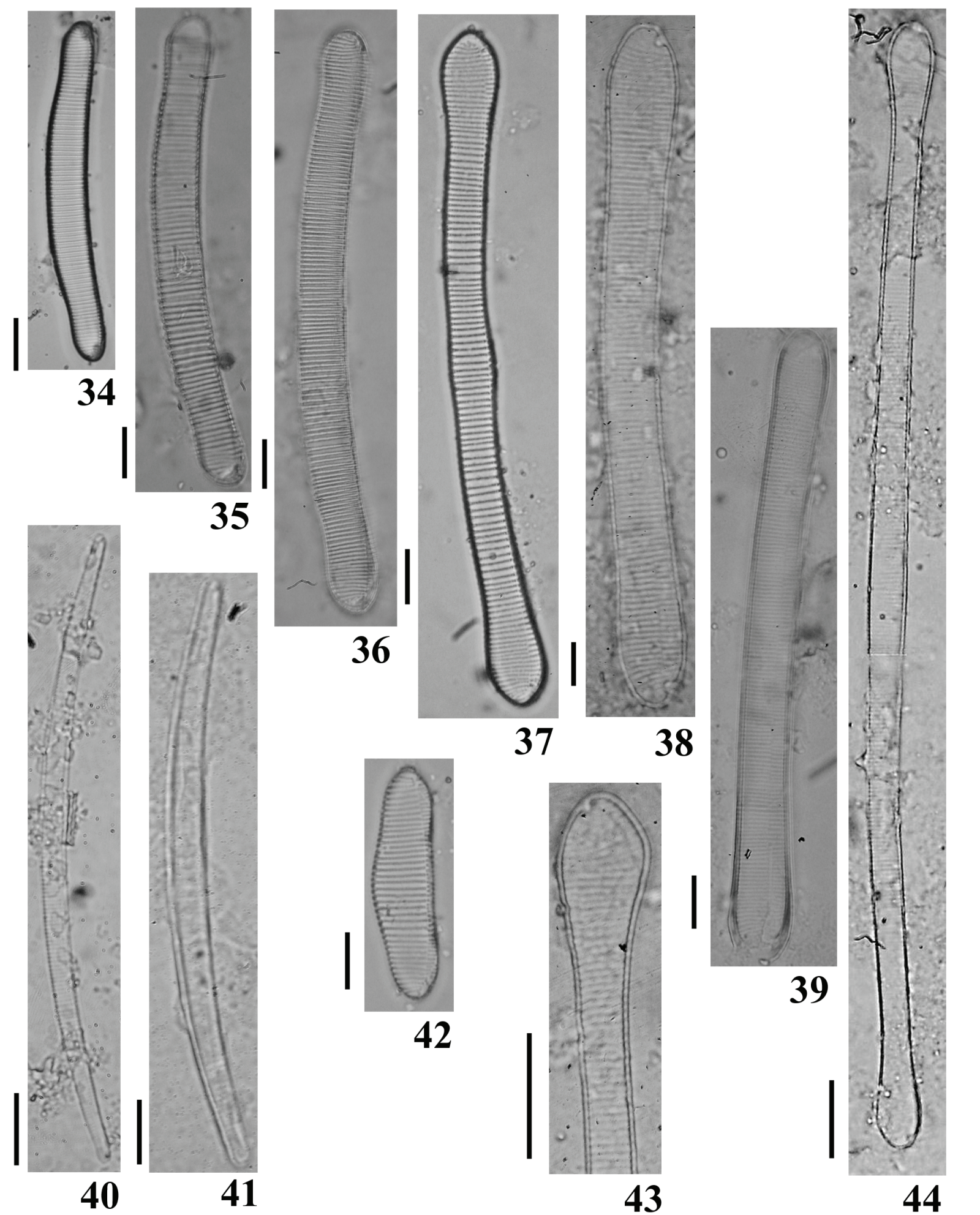


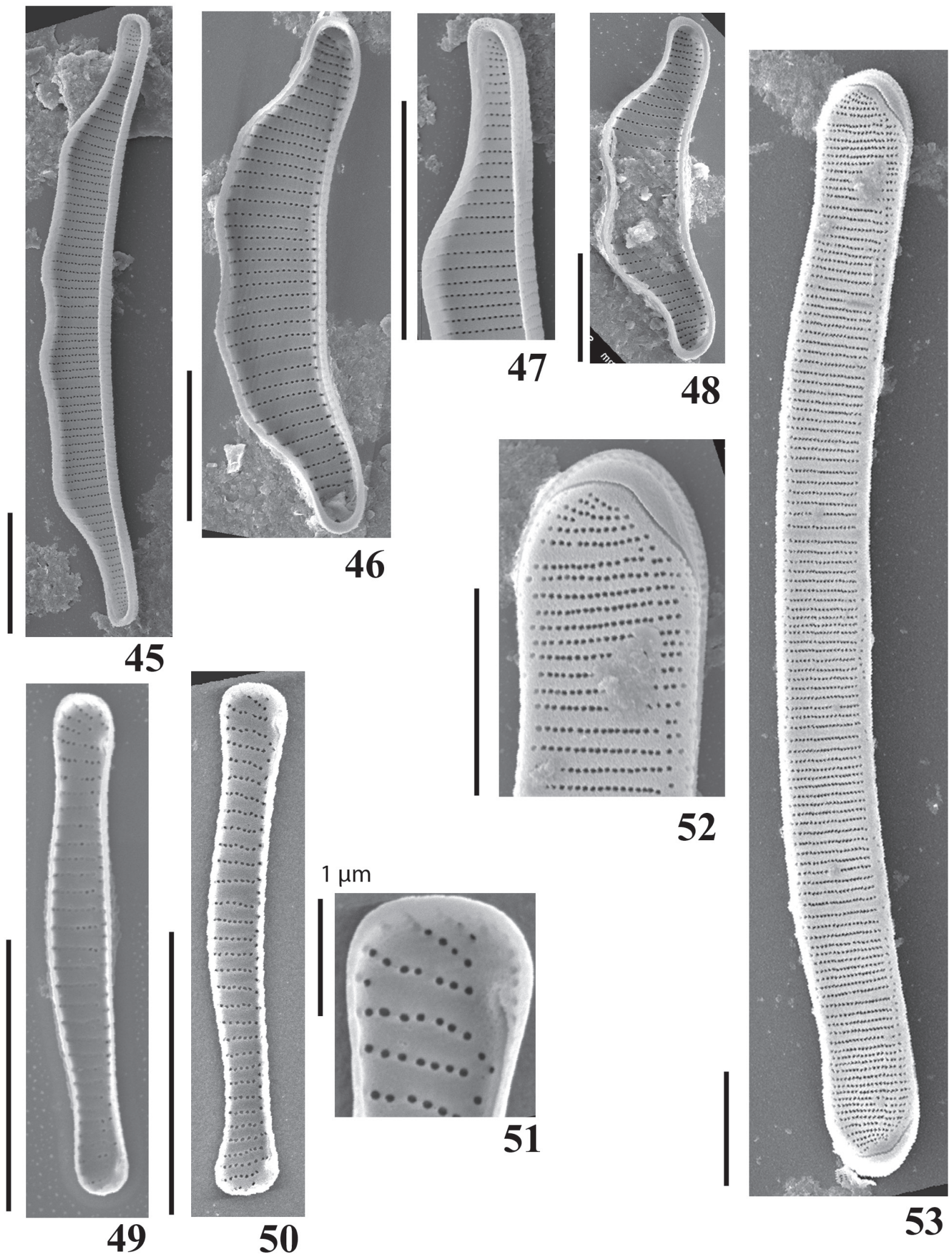

Figuras 45-53. Eunotiaceae Kützing (Bacillariophyceae) planctônicas do Sistema Lago dos Tigres, Britânia, GO, Brasil. 45-47. Eunotia camelus. 48. Eunotia diodon. 49-51. Eunotia asterionelloides. 52-53. Eunotia major. Barras de escala: $10 \mu \mathrm{m}$.

Figures 45-53. Planktonic Eunotiaceae Kützing (Bacillariophyceae) of Sistema Lago dos Tigres, Britânia, Goiás State, Brazil. 45-47. Eunotia camelus. 48. Eunotia diodon. 49-51. Eunotia asterionelloides. 52-53. Eunotia major. Scale bars: $10 \mu \mathrm{m}$. 
Eunotia trigibba Hustedt, A. Schidt's Atlas: pl. 286: figs. 16-18. 1913.

Figura 22

Valvas arqueadas, margem dorsal com três ondulações pronunciadas, margem ventral côncava. Extremidades destacadas do corpo, atenuoarredondadas levemente defletidas dorsalmente. Estrias transapicais convergentes em toda a extensão da valva. Eixo apical: $33-35 \mu \mathrm{m}$; eixo transapical: 6-8 $\mu \mathrm{m}$; 9 estrias em $10 \mu \mathrm{m}$.

Este táxon apresenta contorno valvar semelhante a Eunotia triodon Ehrenberg, no entanto difere pela morfologia da rafe (Metzeltin \& Lange-Bertalot 1998).

Distribuição para a região Centro-Oeste: citação pioneira.

Material examinado: BRASIL. GoIÁs: Britânia, Rio Vermelho, Estação 11, plâncton, 17-X-2004, Nogueira et al. 1033 (UFG29928).

Eunotia tropico-arcus Meltzeltin \& Lange-Bertalot, Iconogr. Diatomol., pl. 74: 1-10. 2007.

Figuras 29-30

Valvas pouco arqueadas, margem dorsal convexa com duas leves ondulações, margem ventral côncava com leve pronunciamento na região mediana, extremidades rostradas. Estrias transapicais paralelas na região mediana e levemente convergente nas extremidades. Eixo apical: 38-59 $\mu \mathrm{m}$; eixo transapical: 7-8 $\mu \mathrm{m}$; 11 estrias em $10 \mu \mathrm{m}$.

Os espécimes analisados assemelham-se ao ilustrado para a espécie em Metzeltin \& LangeBertalot (2007, pág. 414, pr. 74, fig. 9). Esta espécie possui morfologia valvar semelhante a Eunotia bidens Ehrenberg e E. praerupta Ehrenberg, no entanto difere por E. bidens apresentar margem dorsal com maior depressão que E. tropico-arcus, e E. praerupta Ehrenberg possui margem dorsal mais fortemente convexa (Metzeltin \& Lange-Bertalot 2007).

Distribuição para a região Centro-Oeste: citação pioneira.

Material examinado: BRASIL. GoiÁs: Britânia, Lago dos Tigres, Estação 4, plâncton, 4-VI-2004, Nogueira et al. 877 (UFG29866); Lago dos Tigres, Estação 5, plâncton, 4-VI-2004, Nogueira et al. 878 (UFG29867); Lago dos Tigres, Estação 3, plâncton, 19-VIII-2004, Nogueira et al. 945 (UFG29895); Rio Água Limpa, Estação 1, plâncton, 16-X-2004, Nogueira et al. 1023 (UFG29918); Rio Vermelho,
Estação 11, plâncton, 17-X-2004, Nogueira et al. 1033 (UFG29928).

Eunotia zygodon Ehrenberg, Abh. K. Akad. Wiss. Berlin, Physik, Kl. 1841: 415, pl. II: fig. 6. 1843. Figuras 23-24

Valvas com margem dorsal com quatro ondulações, sendo duas na região mediana fortemente pronunciadas e arredondadas, margem ventral côncava, extremidades cuneadas. Estrias transapicais paralelas na região mediana e radiadas nas extremidades, aréolas conspícuas e arredondadas, rafe curta. Nódulos terminais conspícuos nas extremidades na margem ventral. Eixo apical: 77-80 $\mu \mathrm{m}$; eixo transapical: $12-13 \mu \mathrm{m} ; 10$ estrias em $10 \mu \mathrm{m}$.

Este táxon assemelha-se a Eunotia anamargaritae Metzeltin \& Lange-Bertalot, diferem por apresentar ondulações arredondadas ao invés de achatadas presentes em E. anamargaritae. Os espécimes analisados ainda apresentam características próximas a E. yanomami Metzeltin \& Lange-Bertalot, porém diferem quanto a morfologia do nódulo, que em E. zygodon é trilobado (pl. 11, fig. 8, Patrick \& Reimer 1966) e em E. yanomani é simples.

Distribuição para a região Centro-Oeste: Lagoa Bonita (Souza \& Moreira-Filho 1999).

Material examinado: BRASIL. GoIÁs: Britânia, Rio Água Limpa, Estação 1, plâncton, 14-VII-2004, Nogueira et al. 908 (UFG29891).

\section{Agradecimentos}

À Secretaria de Ciência e Tecnologia do Estado de Goiás (SECTEC/GO)/CNPq pelo financiamento do Projeto (Proc. 232340-75); ao Setor de Meteorologia da SECTEC pelos dados metereológicos; à Agência Ambiental do Estado de Goiás por realizar as análises dos nutrientes; à Coordenação de Aperfeiçoamento de Pessoal de Nível Superior (CAPES) pela Bolsa de Mestrado concedida a primeira Autora.

\section{Literatura citada}

Bicca, A.B. \& Torgan, L.C. 2009. Novos registros de Eunotia Ehrenberg (Eunotiaceae-Bacillariophyta) para o Estado do Rio Grande do Sul e Brasil. Acta Botanica Brasilica 23: 427-435.

Bicca, A.B., Torgan, L.C. \& Santos, C.B. 2011. Eunotiaceae (Eunotiales, Bacillariophyta) em ambientes lacustres na Planície Costeira do Sul do Brasil. Revista Brasileira de Botânica 34: 1-19. 
Bicudo, C.E.M. \& Menezes, M. 2006. Gêneros de algas de águas continentais do Brasil (Chave de identificação e descrições). 2 ed. RiMa, São Carlos.

Bicudo, D.C., De-Lamonica-Freire, E.M., Figueiredo, D.M. \& Lima, D. 1995. Ficoflórula do Pantanal de Poconé, Estado de Mato Grosso, Brasil: Centrales e Eunotiaceae (Bacillariophyceae). Hoehnea 22: 165-182.

Bicudo, D.C., Morandi, L.L. \& Ludwig, T.A.V. 1999. Criptógamos do Parque Estadual das Fontes do Ipiranga, São Paulo, SP. Algas, 13: Bacillariophyceae (Eunotiales). Hoehnea 26: 173-184.

Brandão, D. \& Kravchenko, A. 1997. A biota do Campus Samambaia: história, situação e perspectivas. CEGRAF-UFG, Goiânia.

Compère, P. 1975. Algues de la région du lac Tchad, 4: Diatomophycées. Hydrobiologie 9: 203-290.

Contin, L.F. \& Oliveira, R.J.M. 1993. Diatomáceas. In: I.F.P. Campos (coord.). Flora do Estado de Goiás. Criptógamos. v.2, n.1. ABEU/CEGRAF-UFG, Goiânia.

Faria, D.M., Tremarin, P.I. \& Ludwig, T.A.V. 2010. Diatomáceas perifíticas da represa Itaqui, São José dos Pinhais, Paraná: Fragilariales, Eunotiales, Achnanthales e Gomphonema Ehrenberg. Biota Neotropica 10: 415-527.

Ferrari, F., Procopiak, L.K., Alencar, Y.B \& Ludwig, T.A.V. 2007. Eunotiaceae (Bacillariophyceae) em igarapés da Amazônia Central, Manaus e Presidente Figueiredo, Brasil. Acta Amazonica 37: 1-16.

Ferrario, E.M., Sar, E.A. \& Sala, S.E. 1995. Metodologia basica para el estúdio del fitoplancton com especial referencia a las diatomeas. In: K. Alveal, \& M.E. Ferrario (eds.). Manual de métodos ficológicos. Concepción, Universidade de Concepción.

Frenguelli, J. 1933. Diatomeas de la region de los esteros del Yberá. Anales del Museo Nacional de Historia Natural 37: 365-476.

Frenguelli, J. 1941. Diatomeas del rio de La Plata. Revista del Museo de La Plata 3: 213-334.

Schmidt, A. 1874-1959. Atlas der Diatomaceen-Kunde. Leipzing, Reisland, Band III, Serie VI, Tafel 241-288.

Kalff, J. 2002. Limnology: inland water ecosystems. Prentice-Hall, Upper Saddle River.

Kociolek, J.P., Lyon, D. \& Spaulding, S. 2001. Revision of the South American species of Actinella. In: R. Jahn, J.P. Kocioleck, A. Witkowski \& P. Compère (eds.). Studies on diatoms. Gantner Verlag, Koenigstein.

Krammer, K. \& Lange-Bertalot, H. 1998. Bacillariophyceae: Bacillariaceae, Epithemiaceae, Surirellaceae. In: Ettl, H., J. Gerloff, H. Heyning \& D. Mollenhauer (eds.). Süsswasserflora von Mitteleuropa. 2(2). G. Fischer Verlag, Stuttgart.

Lange-Bertalot, H. \& Metzeltin, D. 2009. A dystrophic mountain lake in Panama - Hot spot of new and rare neotropical diatoms. Nova Hedwigia 135: 137-165.
Ludwig, T.A.V. \& Bigunas, P.T.I. 2006. Bacillariophyta. In: C.E.M. Bicudo \& M. Menezes. Gêneros de algas de águas continentais do Brasil (Chave de identificação e descrições). RiMa, São Carlos.

Macedo-Saidah, F.E.M., Nascimento, M.R.R. \& Campos, I.E.P. 1987. O plâncton das águas do Rio Meia Ponte, Município de Goiânia, Goiás, Brasil. Nerítica 2: 105-117.

Medlin, L.K. \& Kaczmarska, L. 2004. Evolution of diatoms: V. Morphological and citological support for the major clades and a taxonomic revision. Phycologia 43: 245-270.

Melo, S., Torgan, L.C. \& Raupp, S.V. 2010. Actinella species (Bacillariophyta) from an Amazon black water foodplain lake (Amazonas - Brazil). Acta Amazonica 40: 269-274.

Metzeltin, D. \& Lange-Bertalot, H. 1998. Tropical Diatoms of South America I. Iconographia Diatomologica. v.5. Koeltz Scientific Books, Stuttgart.

Metzeltin, D., Lange-Bertalot, H. \& García Rodriguez, F. 2005. Diatoms of Uruguay. In: H. Lange-Bertalot (ed.). Iconographia Diatomologica. Annotated diatom micrographs. v.15. Koeltz Scientifc Books, Stuttgart.

Metzeltin, D. \& Lange-Bertalot, H. 2007. Tropical Diatoms of South America II. Special remarks on biogeographic disjunction. In: H. Lange-Bertalot (ed.). Iconographia Diatomologica. Annotated diatom micrographs v.18. Koeltz Scientifc Books, Stuttgart.

Metzeltin, D. \& Tremarin, P.I. 2011. Uma nova espécie de Eunotia para o sudeste do Brasil: Eunotia fuhrmannii. Iheringia Série Botânica 66: 201-208.

Moreira-Filho, H. \& Valente-Moreira, I.M. 1981. Avaliação taxonômica e ecológica das diatomáceas (Bacillariophyceae) epífitas em algas pluricelulares obtidas nos litorais dos Estados do Paraná, Santa Catarina e São Paulo. Boletim Museu Botânico Municipal 47: 1-17.

Nabout, J.C. \& Nogueira, I.S. 2007. Spatial and temporal dynamics of phytoplankton functional group in a blocked valley (Brazil). Acta Limnologica Brasiliensia 19: 305-314.

Patrick, R. 1940. Diatoms of northeastern Brazil. Part I. Coscinodiscaceae, Fragilariaceae and Eunotiaceae. Proceedings of Academy of Natural Sciences of Philadelphia 92: 191-226.

Patrick, R.\& Reimer, C.W. 1966. The diatoms of the United States: exclusive of Alaska and Hawai.1.(Monographs 13). Academy of Natural Sciences, Philadelphia.

Reichardt, E. 1995. Die Diatomeen (Bacillariophyceae) in Ehrenberg's Material von Cayenne, Guyana Gallica (1843). Koeltz Scientific Books. Germany.

Rodrigues, L. \& Moreira Filho, H. 1990. Diatomoflórula do Rio Tubarão, Santa Catarina, Brasil: I - Eunotiaceae Kützing e Achnanthaceae Kützing. Insula 20: 113-135. 
Round, F.E., Crawford, R.M. \& Mann, D.G. 1990. The Diatoms. Biology and Morphology of the genera. Cambridge University Press, United Kindow.

Sabbe, K., Vanhoutte, K., Lowe, R.L., Bergey, E.A., Biggs, B.J.F., Francoeur, S., Hodgson, D. \& Vyverman, W. 2001. Six new Actinella (Bacillariophyta) species from Papua New Guinea, Australia and New Zealand: further evidence for widespread diatom endemism, in the Australasian region. European Journal of Phycology 36: 321-340.

Simonsen, R. 1974. The diatom plankton of the indian ocean expedition of R/V "Meteor". Meteor-forscheingsergeb Reihe D-Biologie 19: 1-66.

Souza, M.G.M. \& Moreira-Filho, H. 1999. Diatoms (Bacillariophyceae) of two aquatic macrophyte banks from Lagoa Bonita, Distrito Federal, Brazil, I: Thalassiosiraceae and Eunotiaceae. Bull Bulletin du Jardin botanique national de Belgique/Bulletin van de nationale Plantentuin van Beige 67: 259-278.
Souza, M.G.M. \& Oliveira, R.I.R. 2007. Levantamento da diatomoflórula epilítica da bacia do rio Paranã, Goiás, Brasil. In: M.J. Martins-Silva (org.). Inventário da biota aquática com vistas à conservação e utilização sustentável do bioma Cerrado (Serra e Vale do rio Paranã). v.1. Ministério do Meio Ambiente, Brasília.

Tremarin, P.I., Ludwig, T.A.V. \& Moreira-Filho, H. 2008. Eunotia Ehrenberg (Bacillariophyceae) do rio Guaraguaçu, litoral do Paraná, Brasil. Acta Botanica Brasilica 22: 845-862.

Van Heurck, H. 1880-1885. Synopsis des diatomées de Belgique. Anver, L'Auteur.

Wetzel, C.E., Ector, L., Hoffmann, L. \& Bicudo, D.C. 2010. Colonial planktonic Eunotia (Bacillariophyceae) from Brazilian Amazon: Taxonomy and biogeographical considerations on the E. asterionelloides species complex. Nova Hedwigia 91: 49-86.

Zalocar deDomitrovic, Y.\& Maidana, N.I. 1997. Taxonomic and ecological studies of the Paraná River diatom flora (Argentina). Heca Diatomologica. J. Cramer, Stuttgart. 
